\title{
NUMERICAL ANALYSIS \\ OF PHOTOENHANCED ELECTRIC CURRENTS IN MOLECULAR MATERIALS
}

\author{
G. Jarosz, R. Signerski and J. Godlewski \\ Department of Molecular Physics, Technical University of Gdańsk \\ Narutowicza 11/12, 80-952 Gdańsk, Poland
}

\begin{abstract}
This paper presents numerical calculations of photoenhanced current-applied voltage and photoenhanced current-light intensity characteristics with regard to the exciton diffusion length, lifetime of excitons and rate of exciton surface quenching. Dependencies of this type cannot be obtained analytically. It is shown that the increase in diffusion length causes the decrease in current in the case of strong surface quenching of excitons. Simultaneously, it is shown that the quality of crystals influences the photoenhanced current. A better quality of crystals causes the increase in the current in the case of weak surface quenching of excitons.
\end{abstract}

PACS numbers: $72.40 .+w$

\section{Introduction}

The photoenhanced current is observed in the space-charge-limited regime due to an optical release of carriers from crystal traps. We consider the photoenhanced current caused by mobile excitons (Frenkel type).

Analytical expressions describing this current can be obtained if:

(i) the optical charge carriers detrapping dominates over the thermal one [1, 2],

(ii) there exists the uniform spatial distribution of excitation or the exponential one $[1,2,3]$.

Usually, the spatial distribution of excitons differs from the exponential one. The exciton concentration depends on the exciton diffusion length, lifetime of excitons and their rate of surface quenching. The purpose of this work is to show the influence of the above-mentioned parameters on the photoenhanced currents. 


\section{Basic equations}

The basic equations describing space-charge-limited currents are as follows:

$$
\begin{aligned}
& j=e p_{\mathrm{f}}(x) \mu F(x), \\
& \varepsilon \varepsilon_{0} \frac{\mathrm{d} F}{\mathrm{~d} x}=\left[p_{\mathrm{f}}(x)+p_{\mathrm{t}}(x)\right] e, \\
& U=\int_{0}^{d} F(x) \mathrm{d} x, \quad F(0)=0,
\end{aligned}
$$

where $j$ is the current density, $p_{\mathrm{f}}$ is the concentration of free charge carriers, $p_{\mathrm{t}}$ is the concentration of trapped charge carriers, $F$ is the electric field strength, $d$ is the sample thickness, $U$ is the applied voltage, $\mu$ is the carrier mobility, and $e, \varepsilon_{0}$ and $\varepsilon$ are the electronic charge, the vacuum permittivity and dielectric constant, respectively.

Using the exponential trap distribution we get the following relation between concentrations of free and trapped carriers [3]:

$$
p_{\mathrm{t}}(x)=p_{\mathrm{f}}(x) \frac{\pi H}{N_{\mathrm{eff}} l \sin (\pi / l)}\left[\eta \frac{\gamma_{\mathrm{Th}} T(x)}{\nu}+\frac{p_{\mathrm{f}}(x)}{N_{\mathrm{eff}}}\right]^{1-1 / l}
$$

with $l=T_{\mathrm{c}} / T$, where $H$ is the total concentration of traps, $T_{\mathrm{c}}$ is the characteristic trap parameter, $k$ is Boltzmann constant, $T$ is absolute temperature, $\nu$ is the thermal collision factor, $\eta$ is the detrapping efficiency, $\gamma_{\mathrm{Th}}$ is the exciton-charge carrier interaction rate constant, $N_{\text {eff }}$ is the effective density of states and $T(x)$ is the exciton concentration.

If we consider the exciton diffusion equation [8]

$$
D_{T} \frac{\mathrm{d}^{2} T(x)}{\mathrm{d} x^{2}}+\alpha \kappa I_{0} \exp (-\kappa x)-k_{T}(x)=0
$$

and quenching of excitons on the both surfaces perpendicular to the current direction in the sample

$$
\left.D_{T} \frac{\mathrm{d} T}{\mathrm{~d} x}\right|_{x=0}=S_{0} T(0),\left.\quad D_{T} \frac{\mathrm{d} T}{\mathrm{~d} x}\right|_{x=d}=-S_{1} T(d),
$$

we get the following formula for the exciton concentration:

$$
\begin{aligned}
T(x) & =\frac{\alpha \kappa I_{0}}{k_{T}\left[1-\left(L_{T} \kappa\right)^{2}\right]}\left[\exp (-\kappa x)+\frac{A \exp \left(x / L_{T}\right)-B \exp \left(-x / L_{T}\right)}{C}\right], \\
A= & \exp \left(-d / L_{T}\right)\left(1+\frac{\kappa L_{T}^{2} k_{T}}{S_{0}}\right)\left(1-\frac{L_{T} k_{T}}{S_{1}}\right) \\
& -\exp (-\kappa d)\left(1-\frac{\kappa L_{T}^{2} k_{T}}{S_{1}}\right)\left(1+\frac{L_{T} k_{T}}{S_{0}}\right) \\
B= & \exp \left(d / L_{T}\right)\left(1+\frac{\kappa L_{T}^{2} k_{T}}{S_{0}}\right)\left(1+\frac{L_{T} k_{T}}{S_{1}}\right) \\
& -\exp (-\kappa d)\left(1-\frac{\kappa L_{T}^{2} k_{T}}{S_{1}}\right)\left(1-\frac{L_{T} k_{T}}{S_{0}}\right)
\end{aligned}
$$




$$
\begin{aligned}
C= & \exp \left(d / L_{T}\right)\left(1+\frac{L_{T} k_{T}}{S_{0}}\right)\left(1+\frac{L_{T} k_{T}}{S_{1}}\right) \\
& -\exp \left(-d / L_{T}\right)\left(1-\frac{L_{T} k_{T}}{S_{0}}\right)\left(1-\frac{L_{T} k_{T}}{S_{1}}\right),
\end{aligned}
$$

where $k_{T}$ is the exciton decay constant, $\kappa$ is the absorption coefficient, $I_{0}$ is the incident light intensity, $\alpha$ is the efficiency of exciton generation, $L_{T}$ is the exciton diffusion length, $D_{T}=L_{T}^{2} k_{T}$ - the diffusion coefficient and $S_{0}, S_{1}$ are the rates of surface quenching of excitons.

\section{Numerical calculation}

In the numerical calculations, based on Eqs.(1)-(7), we want to find the influence of the exciton diffusion length, lifetime of excitons and their surface quenching on photocurrent-applied voltage and photocurrent-light intensity characteristics. As a numerical method we used Euler's method with changeable steps by means of Richardson's approximation [7]. To reduce errors and to increase the speed of the calculations all values were normalized. Values of parameters used to calculation are typical for triplet excitons and traps in molecular materials for a small absorption coefficient of light. For all calculations presented in this work we used: $l=5, H=10^{16} \mathrm{~cm}^{-3}, N_{\mathrm{eff}}=10^{21} \mathrm{~cm}^{-3}[4], \kappa=10^{2} \mathrm{~cm}^{-1}, \nu=10^{12} \mathrm{~s}^{-1}[1,3]$, $\gamma_{\mathrm{Th}}=10^{-11} \mathrm{~cm}^{3} / \mathrm{s}[5,6], k_{T}=10^{2} \mathrm{~s}^{-1}[5], \eta=10^{-2}, \alpha=10^{-2}$.

The photocurrent-applied voltage and photocurrent-light intensity characteristics are illustrated on Figs. 1-4. This characteristics were calculated for thin samples $(5 \mu \mathrm{m})$. For thicker sample the influence of exciton parameters $\left(S_{0}, S_{1}, L_{T}, k_{T}\right)$ on photocurrent is relatively smaller.

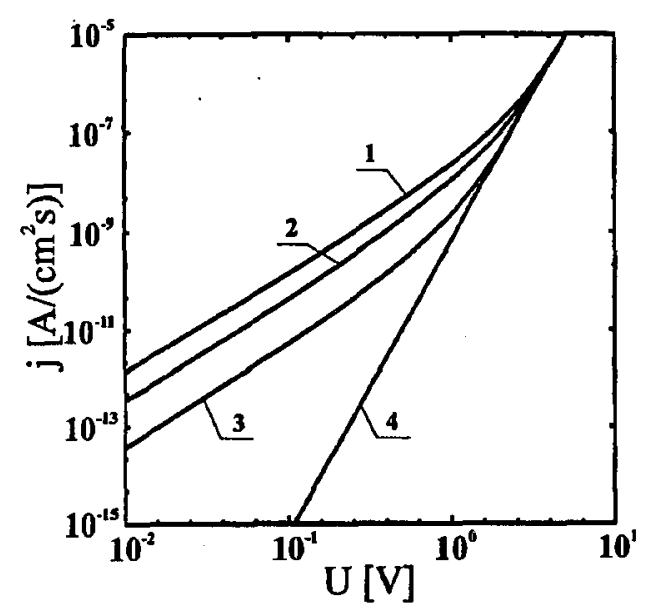

Fig. 1. Photoenhanced current-applied voltage characteristics for different diffusion lengths of excitons in the case of strong exciton surface quenching. (1) $L_{T}=10^{-6} \mathrm{~cm}$, (2) $L_{T}=2 \times 10^{-4} \mathrm{~cm},(3) L_{T}=10^{-3} \mathrm{~cm},(4)$ dark current, $I_{0}=10^{13} 1 /\left(\mathrm{cm}^{2} \mathrm{~s}\right)$. 


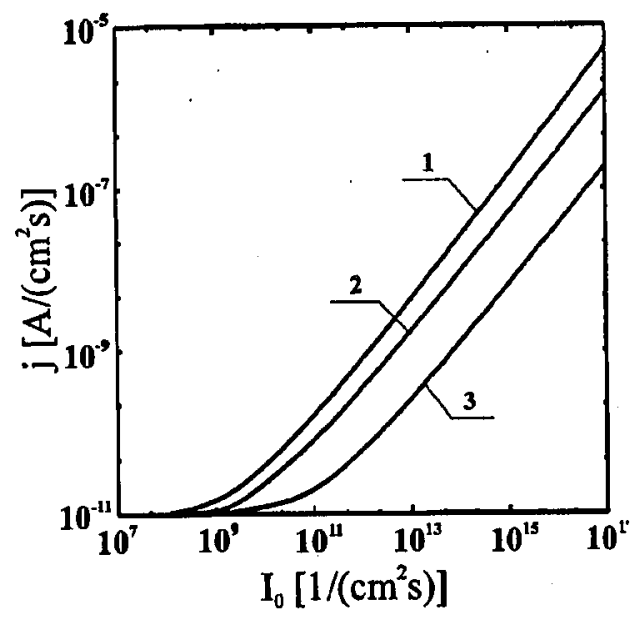

Fig. 2. Photoenhanced current-light intensity characteristics for different diffusion lengths of excitons in the case of strong exciton surface quenching. (1) $L_{T}=10^{-6} \mathrm{~cm}$, (2) $L_{T}=2 \times 10^{-4} \mathrm{~cm},(3) L_{T}=10^{-3} \mathrm{~cm}, U=0.5 \mathrm{~V}$.

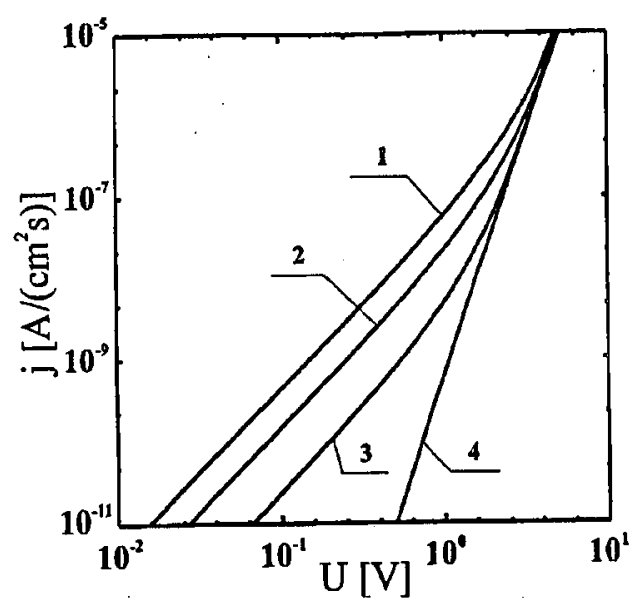

Fig. 3. Photoenhanced current-applied voltage characteristics for different exciton lifetimes in the case of weak exciton surface quenching. (1) $\tau_{T}=40 \mathrm{~ms}$, (2) $\tau_{T}=10 \mathrm{~ms}$, (3) $\tau_{T}=1 \mathrm{~ms}$, (4) dark current. Diffusion coefficient $D_{T}=10^{-4} \mathrm{~cm}^{2} / \mathrm{s}, I_{0}=$ $10^{13} 1 /\left(\mathrm{cm}^{2} \mathrm{~s}\right)$.

In Figs. 1 and 2 characteristics for different exciton diffusion lengths are presented. The characteristics for $L_{T} \rightarrow 0$ correspond to the characteristics for the exponential exciton distribution. Values $L_{T}=2 \mu \mathrm{m}$ and $L_{T}=10 \mu \mathrm{m}$ are adequate to typical triplet exciton diffusion lengths in organic materials like anthracene crystals [5].In the absence of surface quenching of excitons, photocurrent 


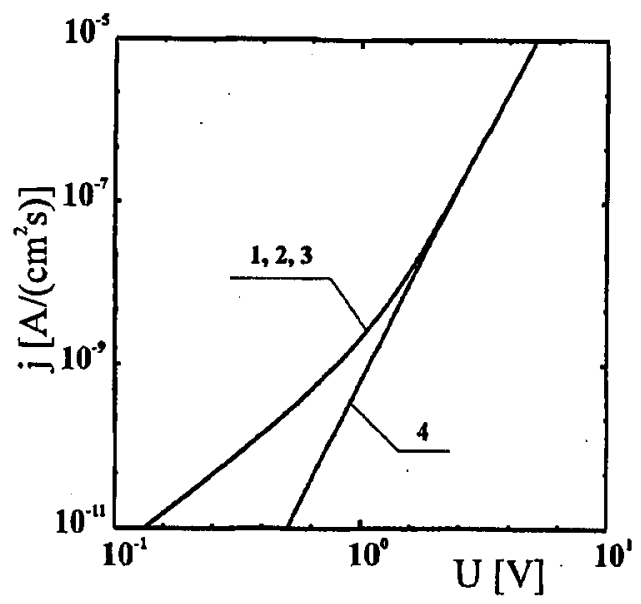

Fig. 4. Photoenhanced current-applied voltage characteristics for different exciton lifetimes in the case of strong exciton surface quenching. (1) $\tau_{T}=40 \mathrm{~ms}$, (2) $\tau_{T}=10 \mathrm{~ms}$, (3) $\tau_{T}=1 \mathrm{~ms}$, (4) dark current. Diffusion coefficient $D_{T}=10^{-4} \mathrm{~cm}^{2} / \mathrm{s} . I_{0}=$ $10^{13} 1 /\left(\mathrm{cm}^{2} \mathrm{~s}\right)$.

characteristics are in agreement with characteristics for the exponential exciton distribution for all values of the exciton diffusion length.

Results presented in Figs. 3 and 4 are connected with the quality of crystals expressed by the exciton lifetime. From Fig. 3 one can notice that for the weak exciton surface quenching the photocurrent is greater for better quality of crystals. In the case of strong exciton surface quenching the photocurrent does not depend on the quality of crystals (Fig. 4).

\section{Conclusions}

The numerical analysis shows the influence of the diffusion length, lifetime and rate surface quenching of excitons on the photoenhanced current. From the numerical analysis we can conclude that:

- for high intensity of incident light the slope of current characteristic is in accordance with the slope of analytical expression $\left(j \propto U^{2}\right.$ and $\left.j \propto I_{0}^{1-1 / l}\right)[2,3]$;

- the increase in the exciton diffusion length causes the decrease in the current in the case of strong surface quenching of excitons;

- a better quality of crystals (higher lifetime of excitons) causes the increase in the photoenhanced current in the case of weak surface quenching of excitons.

However, to understand the phenomenon of the photoenhanced currents in a better way, further numerical studies are needed.

\section{Acknowledgment}

This work was supported in part by the State Committee for Scientific Research (Republic of Poland) project nr 204599101. 


\section{References}

[1] W. Helfrich, Physics and Chemistry of the Organic Solid State, Vol. 3, Eds. D. Fox, M. Labes, A. Weissberger, Interscience Publ., New York, London, Sydney 1967, p. 1.

[2] H. Bauser, H.H. Ruf, Phys. Status Solidi 32, 135 (1969).

[3] J. Godlewski, J. Kalinowski, Phys. Status Solidi A 53, 161 (1979).

[4] W. Helfrich, P. Mark, Z. Phys. 171, 527 (1963).

[5] M. Pope, R. Selsby, Chem. Phys. Lett. 14, 226 (1972).

[6] V. Ern, H. Bouchriha, J. Fourny, G. Delacote, Solid State Comm. 9, 1201 (1971).

[7] G. Dahlquist, Å. Björck, Metody Numeryczne, PWN, Warszawa 1983 (in Polish).

[8] B.I. Mulder, Philips Research Repts., Suppl. 4, 1 (1968). 